Gut, 1989, 30, 1519-1522

Leading article

\title{
Predicting the prognosis of primary biliary cirrhosis
}

There are a number of reasons for attempting to define the prognosis for any patient with primary biliary cirrhosis (PBC). It provides the patient and their family with counselling as to the natural history of the disease and the likely outlook and may also help the physician to determine which patient may be a candidate for specific therapy. Prognostic models have been designed in an attempt to obviate the need for a placebo arm in evaluating therapeutic options, including liver grafting. The observed survival of patients could then be compared with the anticipated survival derived from the estimate of prognosis. The advent of liver replacement as a treatment for end stage disease has made it increasingly important to develop an accurate assessment of prognosis. If the procedure is done too early the patient's life may be prematurely shortened if the operation is unsuccessful, whereas if the procedure is delayed too long then the chances of success will also be considerably reduced.

\section{Asymptomatic primary biliary cirrhosis}

Although asymptomatic primary biliary cirrhosis (disease presenting in the absence of classical 'liver' symptoms) was first described by Fox in 1973, ' it was not until 1980 that it was commonly accepted that the spectrum of PBC was far wider than had previously been thought. ${ }^{2}$. Some patients are diagnosed in the absence of typical symptoms: patients may be detected on routine blood screening for health insurance, after tests for investigation of other symptoms or diseases or by routine screening of blood samples for antimitochondrial antibodies. The increased use of screening and greater awareness of the disease are factors in the three-fold increase in the observed prevalence of asymptomatic patients over a 10 year period. ${ }^{3}$

Asymptomatic patients tend to have a far better prognosis; indeed, some reports suggest that the survival of these asymptomatic patients is not significantly different from that of an age and sex matched general population. ${ }^{4}$ Whether asymptomatic PBC represents a more benign end of the disease or whether asymptomatic disease merely represents a consequence of lead time bias, length bias or selection bias is not yet clear. ${ }^{5}$ As asymptomatic patients tend to be older than symptomatic patients at diagnosis, it seems likely that the asymptomatic patients have a more slowly progressing disease. Once asymptomatic patients become symptomatic, however, their median survival is not dissimilar to that of the main group of patients with $\mathrm{PBC}$ and current figures suggest the median survival of symptomatic patients is about 10 years. 
Within this subgroup of asymptomatic patients, the co-existence of autoimmune disease (such as Sicca syndrome, thyroiditis, Raynaud's disease), hepatomegaly or later histological stage (III/IV) is associated with a more rapid progression. ${ }^{67}$ The presence of portal granulomas, on the diagnostic liver biopsy is associated with a prolonged asymptomatic state. ${ }^{7}$

\section{Symptomatic disease}

It is scarcely surprising that those features of advanced liver disease such as hepatic decompensation are markers of a poor prognosis; thus jaundice, weight loss, ascites, hypoalbuminaemia and histological features of fibrosis and cholestasis are all independently associated with a poor prognosis. Increasing age, at diagnosis, is usually, but not invariably a poor prognostic factor. Less predictably, raised serum IgM and periportal liver cell necrosis are also poor prognostic features. Titre of antimitochondrial antibodies does not correlate with survival. ${ }^{89}$ The histological features which occur in late stage disease indicative of a poor prognosis include cirrhotic transformation, increasing cholestasis, especially with prominant hyaline inclusions, halo formation at the margins of fibrous septa and abundant coarse collagen bundles. Patterns of biliary piecemeal necrosis, and hepatic changes do not correlate with survival. The association with survival of granulomas in the liver in the symptomatic patients remains controversial. ${ }^{11} 1$

In an attempt to give some quantitative estimate of survival attention has focussed more on serological markers because these are more objective and can be more readily quantified.

\section{Biochemical prognostic factors}

Of the standard liver tests, the serum bilirubin is the single most useful prognostic factor in primary biliary cirrhosis. ${ }^{12} 13$ The importance of this factor was emphasised by Shapiro and colleagues in 1979 in a review of 55 patients. The course of disease was divided into two phases: an initial stable period of variable length, when the serum bilirubin remained constant. This was followed by a second period of increasing serum bilirubin (the accelerated phase) culminating in death. The late phase of the disease was characterised by serum bilirubin exceeding $34 \mu \mathrm{mol} / 1$ over a six month period and was associated with a mean survival of 49 months $(90 \%$ confidence limits of 32-74 months). If the bilirubin value exceeded $170 \mu \mathrm{mol} / \mathrm{l}$, survival time was 17 months. These observations have been confirmed by many other studies.

Nevertheless because of the wide variation in confidence limits of serum bilirubin as a prognostic factor, others have attempted to look for more specific markers. Two serological markers have been commended as useful prognostic markers, serum hyaluronate and the N-terminal propeptide of collagen type III (P III P). Neither has yet achieved widespread popularity. Hyaluronic acid, a high molecular weight polysaccharide, is synthesised largely by mesenchymal cells and degraded by liver endothelial cells. In a study of 48 patients followed for $\mathbf{4 0}$ months, Nyberg ${ }^{14}$ showed that serum hyaluronic acid levels increased with progressive histological staging of the disease and correlated significantly with albumin, the galactose tolerance test, serum bilirubin and the prothrombin time. Even in asymptomatic 
patients, the serum hyaluronic acid rose progressively with time. In the 13 patients who died from liver failure during the study the serum hyaluronic acid concentration showed a negative correlation with survival.

$\mathrm{P}$ III $\mathrm{P}$ is a marker of liver fibrosis and concentrations of P III P were studied by Eriksson ${ }^{15}$ in a few patients where the prognostic value correlated with a variety of biochemical and histological features. He reported that $P$ III P concentrations, and serum bilirubin were independent predictions of survival. Both serum bilirubin and P III P at diagnosis could identify those with a poor prognosis. It is not yet clear whether either marker is superior to bilirubin in predicting survival.

Other liver function tests in PBC such as single estimations of aminopyrine, galactosamine or caffeine clearances are of little help, but sequential tests could prove of value.

\section{Prognostic models}

To identify the prognosis for an individual patient more precisely attention has been focussed on the use of models to estimate survival. Three models have been described, all based on the Cox regression analysis, a method which is being used increasingly for analysis of censored data - that is analysis of patients where not all the subjects being studied have reached the survival end point (usually death). Covariation of many predictive values with survival can be used in this analytical model to identify that combination of variables which produces significant results. A prognostic index for a new subject can be obtained by simple mathematical modelling and by use of a graph, the prognostic index can be translated to give either an estimate of the probability of surviving a given time or the estimated median survival time for that individual. There are limitations with the mode $^{16}$ : there is no control for time dependent factors such as the unpredictable nature of life threatening conditions - for example massive variceal haemorrhage, or the removal of adverse factors such as continued alcohol consumption.

The first model was based on 248 patients followed for up to 12 years in the European Multicentre Trial using treatment with azathioprine. ${ }^{17} \mathrm{Six}$ independent variables implied a poor prognosis, of these serum bilirubin was the most significant. The model was validated on independent patient data and correlated well with survival. The Yale model ${ }^{4}$ includes different variables, although again serum bilirubin remains the most significant. The most recent model was developed by the Mayo Clinic using only clinical and laboratory data. ${ }^{18}$ There was good correlation between the European and the Mayo Clinic scores when the same patients were analysed by the different models. Both models have been used to show that liver transplantation is associated with a significant increase in observed survival over that anticipated in the absence of transplantation. ${ }^{19} 20$

\section{Conclusion}

Estimation of survival in patients with primary biliary cirrhosis is now important because of the intervention by liver transplantation. While serum bilirubin is the simplest guide to prognosis the use of models based on Cox regression analysis will give more specific and accurate information, although the formulae and conversion to estimates of survival are still rather 
too cumbersome for 'end of the bed use'. Current models do not take into account time dependent factors and these models only give an estimated probability of survival. No account is taken of the quality of the patient's life, a factor which is vital in the decision for liver transplantation or indeed the success of any other therapeutic intervention. Currently these models do not predict when the unpredictable and life threatening events may occur. Although timing of therapeutic intervention still requires clinical judgement, statistical modelling does at least give some objectivity to aid the clinician.

JAMES M NEUBERGER

The Liver Unit,

The Queen Elizabeth Hospital,

Edgbaston, Birmingham B15 $2 \mathrm{TH}$

\section{References}

1 Fox RA, Scheuer PJ, Sherlock S. Asymptomatic PBC. Gut 1973; 14: 444-7.

2 James O, Macklon AR, Watson AJ. Primary biliary cirrhosis: a revised clinical opinion. Lancet 1981; i: 1278-81.

3 Eriksson S, Lindgren S. The prevalence and clinical spectrum of primary biliary cirrhosis in a population. Scand J Gastroenterol 1984; 19: 971-6.

4 Roll J, Boyer JL, Berry D, Klatskin G. The prognostic importance of clinical and histological features in asymptomatic and symptomatic primary biliary cirrhosis. $N$ Engl $J$ Med 1983; 308: 1-7.

5 Simon JR. Occult blood screening for cholorectal carcinoma - a critical revicw. Gastroenterology 1985; 88: 820-37.

6 Nyberg A, Loof A. Primary biliary cirrhosis: clinical features and outcome with special reference to asymptomatic disease. Scand J Gastroenterol 1989; 24: 57-62.

7 Beswick DR. Klatskin G, Boyer J. Asymptomatic primary biliary cirrhosis. Gastroenterology 1985; 89: 267-71.

8 Christensen E. Crowe J, Doniach D, et al. Clinical pattern and course of disease in primary biliary cirrhosis based on an analysis of 236 patients. Gastroenterology 1980; 78: $236-46$.

9 Klatskin G, Kantor F. Mitochondrial antibodies in primary biliary cirrhosis and other liver discase. Ann Intern Med 1972; 77: 533-41.

10 Portmann B, Popper H, Neuberger J, Williams R. Sequential and diagnostic features in primary biliary cirrhosis based on serial histologic study in 209 patients. Gastroenterology 1985; 88: 1777-90.

11 Lee RG, Epstein O, Jauregui H, Sherlock S, Scheuer PS. Granulomas in primary biliary cirrhosis: a prognostic feature. Gastroenterology 1981: 81: 983-86.

12 Hoffbauer FW. Primary biliary cirrhosis observations in the natural course of the disease in 25 women. Ann J Dig Dis 1960; 5: 348-83.

13 Shapiro JM, Smith H, Schaffner C. Serum bilirubin: a prognostic factor in primary biliary cirrhosis. Gut 1979; 20: 137-40.

14 Nyberg A, Engstrom-Laurent A, Loof L. Scrum hyaluronate in primary biliary cirrhosis a biochemical marker for progressive liver damage. Hepatology 1988; 8: 142-6.

15 Eriksson S, Zettervall O. The N-terminal propeptide of collagen type III in serum as a prognostic indicator in primary biliary cirrhosis. J Hepatol 1982; 2: 370-8.

16 Christensen E. Multivariate survival analysis using Cox's regression model. Hepatology 1987; 7: 1346-58.

17 Christensen E, Neuberger J, Crowe J, et al. Beneficial effect of azathioprine and prediction of prognosis in primary biliary cirrhosis. Gastroenterology 1985; 89: 1084-9.

18 Dickson ER, Grambsch PM, Fleming TR, Fisher LD, Longworthy N. Prognosis in primary biliary cirrhosis: model for decision making. Hepatology 1989; 10: 1-7.

19 Neuberger J, Altman D, Christensen E, Tygstrup N, Williams R. Use of a prognostic index in evaluation of liver transplantation for primary biliary cirrhosis. Transplantation 1980; 41: 713-6.

20 Markus B. Dickson ER, Grambsch P, et al. Efficacy of liver transplantation in patients with primary biliary cirrhosis. $N$ Engl J Med 1989; 320: 1709-13. 